\title{
Filling-In by Joint Interpolation of Vector Fields and Gray Levels
}

\author{
C. Ballester,${ }^{*}$ M. Bertalmio,${ }^{\dagger}$ V. Caselles,${ }^{*}$ G. Sapiro,${ }^{\dagger}$ and J. Verdera,${ }^{*}$
}

April 8, 2000

\begin{abstract}
A variational approach for filling-in regions of missing data in digital images is introduced in this paper. The approach is based on joint interpolation of the image gray-levels and gradient/isophotes directions, smoothly extending in an automatic fashion the isophote lines into the holes of missing data. This interpolation is computed solving the variational problem via its gradient descent flow, which leads to a set of coupled second order partial differential equations, one for the gray-levels and one for the gradient orientations. The process underlying this approach can be considered as an interpretation of the Gestaltist's principle of good continuation. No limitations are imposed on the topology of the holes, and all regions of missing data can be simultaneously processed, even if they are surrounded by completely different structures. Applications of this technique include the restoration of old photographs and removal of superimposed text like dates, subtitles, or publicity. Examples of these applications are given. We conclude the paper with a number of theoretical results on the proposed variational approach and its corresponding gradient descent flow.
\end{abstract}

Keywords: Interpolation, filling-in, image gradients, image gray-levels, variational approach, partial differential equations, Gestalt principles.

\section{Introduction}

Filling-in missing data in digital images has a number of fundamental applications. They range from removing objects from a scene all the way to re-touching damaged paintings and photographs. The basic idea is to fill-in the gap of missing data in a form that it is non-detectable by an ordinary observer. In art, this process is called inpainting [38, 16, 25, 6, 7].

Since the early days of art and photography, filling-in and inpainting has been done by professional artists. Imitating their performance with semi-automatic digital techniques is currently an active area of research, see for example $[23,27,28,8]$ and the papers discussed below. The goal of this work is to introduce a novel algorithm for automatically filling-in gaps in the image. In this article we follow the suggestions in the conclusions section in [6] and introduce an energy

\footnotetext{
${ }^{*}$ Dept. de Tecnologia, University of Pompeu-Fabra, La Rambla 30-32, 08002 Barcelona, Spain, \{coloma.ballester,vicent.caselles,joan.verdera\}@tecn.upf.es

${ }^{\dagger}$ Department of Electrical and Computer Engineering, University of Minnesota, Minneapolis, MN 55455, USA, \{marcelo,guille\}@ece.umn.edu
} 
functional based on an interpretation of Gestaltist's good continuation principle. Suppose that we are given an image $u_{0}: R \rightarrow[a, b]$, where $R$ is a square of $\mathbb{R}^{2}$ and $a<b$, and $\Omega$ is an open bounded subset of $R$ with Lipschitz continuous boundary. We shall call $\Omega$ the hole or gap. We want to fill-in the hole $\Omega$ based on the geometric and photometric information outside the hole. For that we use what we call a band around $\Omega$, i.e., we consider an open region $\tilde{\Omega}$ of $R$ such that $\bar{\Omega} \subseteq \tilde{\Omega}$. The band we refer to will be the set $B=\tilde{\Omega} \backslash \bar{\Omega}$. To fill-in the hole $\Omega$ we use the information of $u_{0}$ contained in $B$, mainly gray level and the vector field of isophotes (level sets) directions of $u_{0}$ in $B$. We attempt to continue the level sets of $u$ in $B$ inside $\Omega$ taking into account the principle of good continuation. We propose an energy functional which takes into account these principles interpreted in a suitable way. The energy functional we propose has to be minimized with respect to two variables : a vector field $\theta$ which represents the directions of the level lines of $u$, and the gray level $u$. $\theta$ and $u$ are constrained in the band $B$ by their known values there. The use of the vector field of directions $\theta$ is one of the main points of the algorithm presented in this paper, which permits the level sets to smoothly continue inside the hole. We are then continuing both the geometric and photometric properties of the image inside the hole.

Let us finally say that the only user interaction required by the algorithm here introduced is to mark the regions to be filled-in. Although a number of techniques exist for the semi-automatic detection of image defects (mainly in films), addressing this is not part of the scope of this paper. Since the algorithm here presented can be used not just to restore damaged photographs but also to remove undesired objects and writings on the image, the regions must be marked by the user, since they depend on his/her subjective selection.

\subsection{Closely related approaches}

Before proceeding with the detailed description of our algorithm, let us comment on related work. Note that image denoising is different to filling-in, since the regions of missing data are usually large. That is, regions occupied by top to bottom scratches along several film frames, long cracks in photographs, superimposed large fonts, and so on, are of significant larger size than the type of noise assumed in common image enhancement algorithms. In addition, in common image enhancement applications, the pixels contain both information about the real data and the noise (e.g., image plus noise for additive noise), while in our application there is no significant information in the region to be inpainted.

A very active area related to the work here presented is the restoration of damaged films. The basic idea here is to use information from past and future frames to restore the current one, e.g., $[23,28]$. Of course, this general approach can not be used when dealing with still images.

Another area related to the work here described is texture synthesis. The basic idea here is to select a texture and synthesize it inside the region to be filled-in (the hole). Although

outstanding texture synthesis results have been reported in the literature, e.g., [22, 14, 19, 35], these algorithms require the user to select the texture to be copied into the hole. For images where the region to be replaced covers several different structures, the user would need to go through the tremendous work of segmenting them and searching corresponding replacements throughout the picture. Although part of this search can be done automatically, this is extremely 
time consuming and requires the non-trivial selection of many critical parameters, e.g., [14].

Last, a number of fundamental works on disocclusion and line continuation have been reported in the literature, and these are the closest to our approach. A pioneering contribution in this area is described in [33]. The authors presented a technique for removing occlusions with the goal of image segmentation. Since the region to be filled-in can be considered as occluding objects, removing occlusions is analogous to image inpainting. The basic idea suggested by the authors is to connect T-junctions at the same gray-level with elastica minimizing curves (see later in this paper for the exact definition of elastica curves). The technique was mainly developed for simple images, with only a few objects with constant gray-levels, and will not be applicable for the examples with natural images presented later in this paper. (Other researchers, e.g., D. Jacobs, R. Basri, S. Zucker, etc, have followed this interesting research area, mainly developing techniques for smooth curve continuation.) Masnou and Morel [31, 32] recently extended these ideas, presenting a very elegant and inspiring general formal variational formulation for disocclusion and a particular practical algorithm implementing some of the ideas in this formulation. The algorithm fills-in by joining with geodesic curves the points of the isophotes arriving at the boundary of the region to be inpainted. The holes in their algorithm are limited to having simple topology. In addition, the angle with which the level lines arrive at the boundary of the holes are not (well) preserved, and the algorithm uses straight lines to join equal gray value pixels. On the other hand, we should note that this work has motivated in part and inspired our work.

Recently, we have addressed the concept of smooth continuation of information in the levellines direction in [6, 7]. We proposed an algorithm, inspired in partial differential equations, that propagates the image Laplacian in this direction. The algorithm attempts to imitate basic approaches used by professional restorators. The algorithm also introduced the importance of propagating both the gradient direction (geometry) and gray-values (photometry) of the image in a band surrounding the hole to be filled-in. It is part of the goal of the current paper to adopt some of the ideas of $[6,7]$, while deviating from the particular model in order to be able to define a formal variational approach to the filling-in problem.

The work in $[6,7]$ inspired a very elegant approach to the filling-in problem recently reported in [9] (this work was performed independently to the one reported in this paper). ${ }^{1}$ The authors present a clear and very intuitive axiomatic approach to the problem. The main algorithm they propose after an interesting discussion of the inpainting problem is to minimize the Total Variation (TV), [34], of the image inside the hole (they also use, as proposed in $[6,7]$ and further studied in this paper, a band surrounding the region). They address in addition the interpolation and filling-in in the presence of noise, a very important additional contribution. As in the work of Masnou and Morel, their interpolation is limited to creating straight isophotes, not necessarily smoothly continued from the hole boundary, and mainly is developed (as the authors clearly state) for small holes. Although straight connections give visually pleasant results for small holes, it is important to develop a theory that permits interpolation of level lines across large gaps. As we will argue later in this paper, in order to obtain such a smooth interpolation of isophotes, it is necessary to go into high order PDE's or systems of PDE's, as done in [6, 7] and

\footnotetext{
${ }^{1}$ We thank the authors for providing us with a preliminary report of their work.
} 
here.

To conclude this section, the interested reader is referred to the works of Nitzberg-MumfordShiota, Masnou-Morel, and Chan-Shen (as well as our previous work) to study other interesting and very related techniques for filling-in.

Let us conclude with the plan of the paper. In Section 2 we introduce the problem, the functional spaces and the energy functional for image inpainting. To clarify the meaning of the functional, we also discuss the particular case where we interpolate the gray level, knowing the vector field of directions. Section 3 is devoted to numerical experiments. Section 4 contains some conclusions. Finally, the Appendix is devoted to the proof of existence of minimizers for the energy functional introduced in this paper.

\section{Joint interpolation of vector fields and gray values}

Let $u_{0}: R \rightarrow \mathbb{R}$ be an image defined on a domain $R$ of $\mathbb{R}^{2}$, which we may suppose to be a square. Let $\Omega, \tilde{\Omega}$ be two open bounded domains in $\mathbb{R}^{2}$ with Lipschitz boundary and suppose that $\bar{\Omega} \subseteq \tilde{\Omega} \subset \subset R$. To simplify our presentation we shall assume that $\tilde{\Omega}$ does not touch the boundary of the image domain $R$. Let $B:=\tilde{\Omega} \backslash \bar{\Omega}$. $B$ will be called the band around $\Omega$. Suppose that a function $u_{0}$ is given in $B$ which, for the moment being, we shall assume to be smooth in $\bar{B}$ (later we shall assume that $u_{0}$ is of bounded variation, i.e., $u_{0} \in B V(B)$ ). Let $\theta_{0}$ be the vector field of directions of the gradient of $u_{0}$ on $B$, i.e., $\theta_{0}$ is a vector field with values in $\mathbb{R}^{2}$ satisfying $\theta_{0}(x) \cdot \nabla u_{0}(x)=\left|\nabla u_{0}(x)\right|$ and $\left|\theta_{0}(x)\right| \leq 1$ (ideally 1 or 0 , see below). This is the information we shall use on $B$.

We pose the image inpainting problem in the following form: Can we extend (in a reasonable way) the pair of functions $\left(u_{0}, \theta_{0}\right)$ from the band $\tilde{\Omega} \backslash \bar{\Omega}$ to a pair of functions $(u, \theta)$ defined inside $\Omega$ ? Of course, we will have to precise what we mean by a reasonable way. We shall discuss and analyze a variational formulation of this filling-in problem and discuss possible energy functionals, and their corresponding gradient descent flows, which give a solution to it. The data are given on the band $B$ and we should constraint the solution $(u, \theta)$ to be near the data on $B$. The vector field $\theta$ should satisfy $|\theta| \leq 1$ on $\Omega$ and should be related to $u$ by trying to impose that $\theta \cdot \nabla u=|\nabla u|$, i.e., we should impose that $\theta$ is related to the vector field of directions of the gradient of $u$. The condition $|\theta(x)| \leq 1$ should be interpreted as a relaxation of this. Indeed, it may happen that $\theta(x)=0$ (flat regions) and then we cannot normalize the vector field to a unit vector. We should have in mind that the ideal case would be that $\theta=\frac{\nabla u}{\nabla u}$, $u$ being a smooth function with $\nabla u(x) \neq 0$ for all $x \in \Omega$. Finally, we should impose that the vector field $\theta_{0}$ in the band tries to continue smoothly to $\theta$ inside $\Omega$. We shall impose this by observing that, in case $\theta$ represents the directions of the normals to the level lines of $u$, i.e., of the curves $u\left(x_{1}, x_{2}\right)=\lambda, \lambda \in \mathbb{R}$, then a term like $\operatorname{div}(\theta)$ represents its curvature. Motivated by the principle of smooth continuation, our energy functional should contain terms integrating $\operatorname{div}(\theta)$. Indeed, collecting all the observations above, we propose to minimize a functional of the form

$$
\operatorname{Minimize} \int_{\tilde{\Omega}}|\operatorname{div}(\theta)|^{p}(a+b|\nabla k * u|) d x+\alpha \int_{\tilde{\Omega}}(|\nabla u|-\theta \cdot \nabla u) d x,
$$


where $a, b$, and $\alpha$ are positive constants and $k$ is a smoothing kernel. We need to give a sense to the integrals appearing in the above expression and to make precise the admissible class of functions where the functional has to be minimized. For that, we need to introduce some function spaces. This is done in the following section. Once this has been formally addressed, we will further discuss the underlying concepts of the above functional.

\section{$2.1 \quad$ Function spaces}

Let us first recall the definition of $B V$ functions and total variation. Let $Q$ be an open set. A function $u \in L^{1}(Q)$ whose partial derivatives in the sense of distributions are measures with finite total variation in $Q$ is called a function of bounded variation. The class of such functions will be denoted by $B V(Q)$. Thus $u \in B V(Q)$ if and only if there are Radon measures $\mu_{1}, \ldots, \mu_{N}$ defined in $Q$ with finite total mass in $Q$ and

$$
\int_{Q} u D_{i} \varphi d x=-\int_{Q} \varphi d \mu_{i}
$$

for all $\varphi \in C_{0}^{\infty}(Q), i=1, \ldots, N$. Thus the gradient of $u$ is a vector valued measure with finite total variation

$$
\|\nabla u\|=\sup \left\{\int_{Q} u \operatorname{div} \varphi d x: \varphi \in C_{0}^{\infty}\left(Q, \mathbb{R}^{n}\right),|\varphi(x)| \leq 1 \text { for } x \in Q\right\} .
$$

The space $B V(Q)$ is endowed with the norm

$$
\|u\|_{B V}=\|u\|_{L^{1}(Q)}+\|\nabla u\| .
$$

We say that a measurable set $E \subseteq Q$ has finite perimeter in $Q$ if its indicator function $\chi_{E} \in$ $B V(Q)$. If $u \in B V(Q)$ almost all its level sets $[u \geq \lambda]=\{x \in Q: u(x) \geq \lambda\}$ are sets of finite perimeter. For sets of finite perimeter $E$ one can define the essential boundary $\partial^{*} E$, which is rectifable with finite $H^{N-1}$ measure, and compute the normal to the level set at $H^{N-1}$ almost all points of $\partial^{*} E$. Thus at almost all points of almost all level sets of $u \in B V(Q)$ we may define a normal vector $\theta(x)$. This vector field of normals $\theta$ can be also defined (hence extended to all $Q)$ as the Radon-Nikodym derivative of the measure $\nabla u$ with respect to $|\nabla u|$, i.e., it formally satisfies $\theta \cdot \nabla u=|\nabla u|$ and, also, $|\theta| \leq 1$ a.e.. For further information concerning functions of bounded variation we refer to $[1,17,39]$.

Let us now introduce the function spaces for $\theta$. Let $Q$ be an open bounded subset of $\mathbb{R}^{2}$ with a Lipschitz boundary. We define

$$
W^{1, p}(\operatorname{div}, Q)=\left\{\theta \in L^{p}(Q)^{2}: \operatorname{div}(\theta) \in L^{p}(Q)\right\}, \quad 1 \leq p<\infty
$$

and

$$
M(\operatorname{div}, Q)=\left\{\theta \in L^{1}(Q)^{2}: \operatorname{div}(\theta) \text { is a Radon measure in } Q\right\} .
$$

The Trace Theorem ([2],[10]) guarantees that the normal component $\left.\theta \cdot n\right|_{\partial Q}$, is well defined for vector fields $\theta$ in $W^{1, p}(\operatorname{div}, Q)$, or in $M(\operatorname{div}, Q)$. To simplify our notation we shall assume that $W^{1,1}(\operatorname{div}, Q)$ represents the space $M(\operatorname{div}, Q)$. 
Next, we shall give a sense to the integrals of bounded vector fields with divergence in $L^{p}$ integrated with respect to the gradient of a $B V$ function. For that, we shall need some results from [2] (see also [26] and [11]). Let $Q$ be an open bounded subset of $\mathbb{R}^{n}$ with Lipschitz continuous boundary. Let $p \geq 1$ and $q \geq 1$ be such that $\frac{1}{p}+\frac{1}{q}=1$. Following [2], let

$$
X(Q)_{p}=\left\{z \in L^{\infty}\left(Q, \mathbb{R}^{n}\right): \operatorname{div}(z) \in L^{p}(Q)\right\}
$$

If $z \in X(Q)_{p}$ and $w \in B V(Q) \cap L^{q}(Q)$ we define the functional $(z, \nabla w): C_{0}^{\infty}(Q) \rightarrow \mathbb{R}$ by the formula

$$
<(z, \nabla w), \varphi>=-\int_{Q} w \varphi \operatorname{div}(z) d x-\int_{Q} w z \cdot \nabla \varphi d x .
$$

Then $(z, \nabla w)$ is a Radon measure in $Q$,

$$
\int_{Q}(z, \nabla w)=\int_{Q} z \cdot \nabla w d x
$$

for all $w \in W^{1,1}(Q) \cap L^{p}(Q)$ and

$$
\left|\int_{B}(z, \nabla w)\right| \leq \int_{B}|(z, \nabla w)| \leq\|z\|_{\infty} \int_{B}\|\nabla w\|
$$

for any Borel set $B \subseteq Q$.

In [2], a weak trace on $\partial Q$ of the normal component of $z \in X(Q)_{p}$ is defined. Concretely, it is proved that there exists a linear operator $\gamma: X(Q)_{p} \rightarrow L^{\infty}(\partial Q)$ such that

$$
\begin{gathered}
\|\gamma(z)\|_{\infty} \leq\|z\|_{\infty} \\
\gamma(z)(x)=z(x) \cdot \nu(x) \quad \text { for all } x \in \partial Q \text { if } z \in C^{1}\left(\bar{Q}, \mathbb{R}^{N}\right)
\end{gathered}
$$

We shall denote $\gamma(z)(x)$ by $[z, \nu](x)$. Moreover, the following Green's formula, relating the function $[z, \nu]$ and the measure $(z, \nabla w)$, for $z \in X(Q)_{p}$ and $w \in B V(Q) \cap L^{q}(Q)$, is established:

$$
\int_{Q} w \operatorname{div}(z) d x+\int_{Q}(z, \nabla w)=\int_{\partial Q}[z, \nu] w d H^{N-1}
$$

If no confusion arises, we shall denote $z \cdot \nabla w$ instead of $(z, \nabla u)$ for $z \in X(Q)_{p}, w \in B V(Q) \cap L^{q}(Q)$

\subsection{The energy derivation and interpretation}

One of the key concepts above was the band around the hole. The band is of local character but in principle it could be extended to all the known part of the image. Obviously, what happens at distant parts can be independent or not from what happens at the hole, but, in our construction below, we suppose that only a narrow band around the hole influences what happens inside the hole. Could we fill-in without the band ? To discuss this suppose that we are given the image of Figure 1.a which is a gray band on a black background partially occluded by a square $\Omega$. We suppose that the sides of the square hole $\Omega$ are orthogonal to the level lines of the original image. In these conditions, the normal component of the vector field $\theta_{0}$ outside $\Omega$ is null at $\partial \Omega$. Thus if the boundary data is just $\left.\theta_{0} \cdot n\right|_{\partial \Omega}$, we would have that $\left.\theta_{0} \cdot n\right|_{\partial \Omega}=0$. In particular, the vector 
field $\theta=0$ satisfies this condition. If we are not able to propagate $\theta$ inside $\Omega$ this may become an unpleasant situation, since this would mean that we do no propagate the values of $u$ at the boundary. If we write the functional (1) with $\theta=0$, it turns out to be the Total Variation [34]. The decision of extending the gray band or filling the hole with the black level would be taken as a function of the perimeter of the discontinuities of the function in the hole. To overcome this situation we introduce the band which ensures that the vector field outside $\Omega$ is present in the functional. In Figure 1.b we display the result of the interpolation with $\theta=0$ on $\Omega$. In Figure 1.c we display the result of the interpolation using the functional we shall completely describe below, which takes into account the band $B$ and computes the vector field $\theta$ in $\tilde{\Omega}=\bar{\Omega} \cup B$.

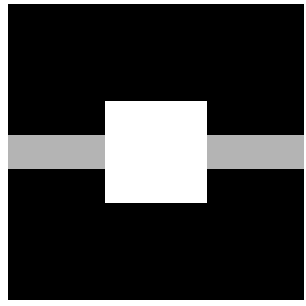

Figure 1.a.

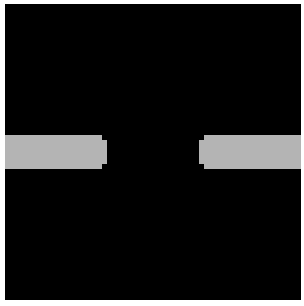

Figure 1.b.

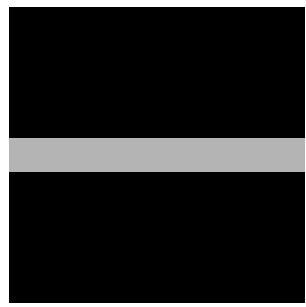

Figure 1.c.

Thus, let $B$ be a band around $\Omega$ with a Lipschitz boundary containing the boundary of $\Omega$ (see Figure 2). As we made explicit above, $B=\tilde{\Omega} \backslash \bar{\Omega}$. Given the band $B$ and the function $u_{0}$ of bounded variation in $B$, we define the space

$$
B V\left(\tilde{\Omega}, B, u_{0}\right)=\left\{u \in B V(\tilde{\Omega}): u=u_{0} \text { in } B\right\} .
$$

Let $\theta_{0}: B \rightarrow \mathbb{R}^{2}$ be a vector field of directions of the gradient of $u_{0}$, i.e., $\left|\theta_{0}\right| \leq 1$ and $\theta_{0} \cdot \nabla u_{0}=\left|\nabla u_{0}\right|$ a.e. in $B$. In practice we shall constraint the vector field $\theta$ to be the vector field of directions of $u_{0}$ only indirectly, through the functional. We could also introduce this as a constraint or with a penalty term $\int_{B}\left(\theta-\theta_{0}\right)^{2}$ (see also $[9,34]$ for penalties of this form in the gray values).

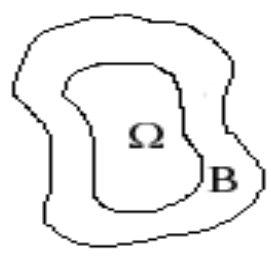

Figure 2. 
Combining the previous elements, the band, the relations between $\theta$ and $u$, and the smoothness term on $\theta$, we propose to interpolate the pair $(\theta, u)$ in $\Omega$ by minimizing the functional:

$$
\begin{aligned}
& \text { Minimize } \int_{\tilde{\Omega}}|\operatorname{div}(\theta)|^{p}(a+b|\nabla k * u|) d x+\alpha \int_{\tilde{\Omega}}(|\nabla u|-\theta \cdot \nabla u) d x \\
& \theta \in W^{1, p}(\operatorname{div}, \tilde{\Omega}) \\
& u \in B V\left(\tilde{\Omega}, B, u_{0}\right) \\
& \left.\theta \cdot n\right|_{\partial \tilde{\Omega}}=\left.\theta_{0} \cdot n\right|_{\partial \tilde{\Omega}} \\
& |\theta| \leq 1 \\
& |u| \leq\left\|u_{0}\right\|_{L^{\infty}(B)} .
\end{aligned}
$$

where $a, \alpha>0, b \geq 0$ and $k$ denotes a regularizing kernel of class $C^{1}$ such that $k(x)>0$ a.e.. The previous functional is coercive and admits a minimum in the class of functions described above if $p>1$. The case $p=1$ is under study. The functional can be interpreted as a formulation of the principle of good continuation and amodal completion as formulated in the Gestalt theory of vision. The following remarks contain heuristic arguments which may help to understand our choice. In next subsection we shall explain in more detail the role of the term coupling $\theta$ and $u$.

\section{Remarks.}

1. The constant $b$ is $\geq 0$. If $u$ is the characteristic function of the region enclosed by a curve $C$ then a term like

$$
\int_{\tilde{\Omega}}|\operatorname{div}(\theta)|^{p}|\nabla u|
$$

is related to $\int_{C}|\kappa|^{p} d s$, where $\kappa$ is the Euclidean curvature (of the level-sets). If $p=2$, this term appears in Euler's elastica,

$$
\int_{C}\left(\alpha+\beta \kappa^{2}\right) d s, \quad \alpha, \beta>0 .
$$

Euler's elastica (12) was proposed in [33] as a technique for removing occlusions with the goal of image segmentation, since this criterion yields smooth, short, and not too curvy curves. In terms of characteristic functions, Euler's elastica can be written as

$$
\int|\nabla u|\left(\alpha+\beta\left|\operatorname{div}\left(\frac{\nabla u}{|\nabla u|}\right)\right|\right)^{2} \text {. }
$$

In [5], it was shown that this functional is not lower semicontinuous. The functional proposed by Masnou and Morel [31], [32] can be interpreted as a relaxation of it, since it integrates functionals like the elastica (plus the angle that the curve makes with the corresponding level line arriving at the boundary) along the level lines of the function $u$. Our functional can be also considered as a relaxed formulation of the energy of the elastica. For that, we introduced $\theta$ as a independent variable, and we tried to couple it to $u$ with the term

$$
\int_{\tilde{\Omega}}|\nabla u|-\theta \cdot \nabla u
$$

so that, heuristically, we try to impose that $\theta \cdot \nabla u=|\nabla u|$ (see next Subsection for a detailed discussion of this term). Finally, let us say that for mathematical reasons we have 
convolved the $\nabla u$ term of (11) to be able to prove the existence of a minimum for (10). From a theoretical point of view, this may invalidate our previous comments. But, from a practical point of view, it gives a weight to the curve of discontinuities of the image.

2. The constant $a$ has to be $>0$. Otherwise we do not get compactness on $\theta$. Now, let us comment on the two terms containing $\operatorname{div}(\theta)$. Heuristically, if we do not compute $\theta$ in a proper way, in a continuous image like in Figure 1, $\theta$ could be zero except on a set of curves. Then $\theta=0$ a.e. on $B$ (or on $\tilde{\Omega}$ ) and a term like

$$
\int_{\tilde{\Omega}}|\operatorname{div}(\theta)|^{p} d x
$$

would produce a null value since $\operatorname{div}(\theta)=0$. On the other hand, a term like (11) would integrate a power of the curvature on the level line corresponding to the discontinuity of the image and it would guarantee that the functional is not null. This argument is only heuristic and not completely justified. Indeed, we believe that in such example as in Figure 1 , a term like (15) would induce a regularizing effect on $\theta$ and the support of $\theta$ would not be a curve any more. In that case, the integral (15) would not be null.

3. Related to the question discussed in the last comment is the possibility to compute a regularized vector field of directions for images which are constant except at jump discontinuities. A direct computation of the vector field outside the hole in an image like Figure 1.a gives a null vector field at all points except the points on the level line separating the black from the white region. This may be not be a good starting point to extend reasonably the vector field $\theta$ inside $\Omega$. To initialize the algorithm of steepest descent, a regularization of $\theta$ outside $\Omega$ may be constructed as the vector field of directions of the image $U_{0}(t, x)$ obtained by regularizing $u_{0}(x), x \in B$, with the equation

$$
\begin{array}{ll}
\frac{\partial u}{\partial t}=\operatorname{div}\left(\frac{\nabla u}{|\nabla u|}\right) & \text { in } Q=(0, \infty) \times B \\
\frac{\partial u}{\partial \eta}=0 & \text { in } S=(0, \infty) \times \partial B \\
u(0, x)=u_{0}(x) & \text { for } x \in B .
\end{array}
$$

As it is shown in [3], this equation permits a regularization of the vector field of directions of the gradient of $u$, i.e., there is a vector field $z,|z| \leq 1$, such that $u_{t}=\operatorname{div}(z)$ and $\int_{B} z \cdot \nabla u=\int_{B}|\nabla u|$. Moreover, for each $t>0, \operatorname{div}(z(t)) \in L^{p}(B)$ if $u_{0} \in L^{p}(B)$ for all $p \geq 1$. In this way, we initialize the steepest descent algorithm described in Section 3 with a regularized vector field $\theta$. This again raises a question, namely, if this ad-hoc regularization is really needed or a regularization takes place with the algorithm itself, if we use an implicit numerical scheme to solve (10).

4. The bound $|u| \leq\left\|u_{0}\right\|_{L^{\infty}(B)}$ can be replaced by a constant depending on $\left\|u_{0}\right\|_{L^{\infty}(B)}$. The constraint that $u=u_{0}$ in $B$ could be relaxed by adding a penalty term like $\int_{B}\left(u-u_{0}\right)^{2}$. 
Similarly, we could add a penalty term to constraint $\theta$ to be near $\theta_{0}$ inside $B$. In this case, we should regularize $\theta_{0}$ in $B$ using the equation described in Remark 3 . This type of approach is addressed in the work of Chan and Shen mentioned before [9].

5. In practice, functional (10) is used to interpolate shapes, i.e., to interpolate level sets. The image is decomposed into upper level sets $\left[u_{0} \geq \lambda\right]$, which are interpolated using (10) to produce the level sets $X_{\lambda} u$ of the function $u$ which reconstructed inside $\Omega$ by using the reconstruction formula

$$
u(x)=\sup \left\{\lambda: x \in X_{\lambda} u\right\} .
$$

In principle, (10) could be used directly to interpolate functions. But, discontinuities of the image have a contribution to the energy which is proportional to the jump. This gives different weights to discontinuities of different sizes and, as a consequence, they are not treated in the same manner. This is not reasonable if we want to interpolate the shapes of the image, independently of their contrast. When taking level sets, we treat all shapes equally, and the parameters of the functional should only weight geometric quantities (like length, total curvature) and decide which interpolation is taken as a function of them. Which are the precise geometric quantities is not known precisely. The functional was introduced on a heuristic basis, but relaxations may occur as they occur in (16), where $\operatorname{div}\left(\frac{\nabla u}{\nabla u}\right)$ may represent $\frac{\text { perimeter }}{\text { area }}$ when computed on a flat region $([3,36])$. This requires further study and we shall pursue it elsewhere.

6. The choice made in Remark 5 of decomposing the image $u_{0}$ into upper level sets, interpolating them and reconstructing the function $u$, introduces a lack of symmetry. Indeed, we are giving more weight to upper level sets than to lower level sets. This can be seen in Figure 3. Figure 3.a displays the image to be interpolated. It is clear that several reasonable solutions are possible and no one of them is preferable to the others. The choice we made gives Figure 3.b as solution, favoring that the object whose level is 210 goes above the object whose level is 0 . But, in that case, the "true" information is lacking and we selected one of the possible reasonable solution.

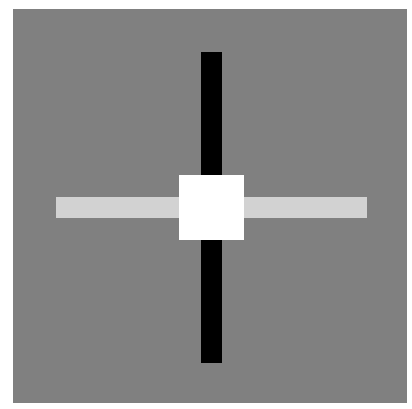

Figure 3.a.

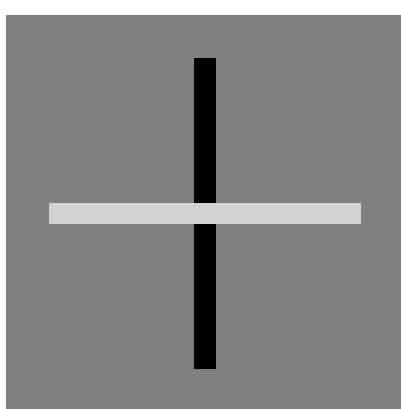

Figure 3.b. 


\subsection{Interpolating gray values along the integral curves of a vector field}

Our purpose in this section is to further discuss the term

$$
\int_{\tilde{\Omega}}|\nabla u|-\theta \cdot \nabla u
$$

in functional (10) (see also [24] for a related, $L_{2}$ and Poisson-equation based, approach of gray value reconstruction from image gradients). We shall see that (when $\theta$ is known), when minimizing (17), we are constructing the function $u$ whose values on the boundary are given and whose direction of the gradient is given by $\theta$. We shall discuss this from a general point of view. Thus, suppose that $\Omega$ is an open bounded domain with a Lipschitz boundary and $\varphi \in L^{\infty}(\partial \Omega)$. Let $\nu: \Omega \rightarrow \mathbb{R}^{2}$ be a vector field whose smoothness will be detailed below. We ask the following question: can we interpolate the boundary data $\varphi$ along the integral curves of $\nu$ ? In the case discussed in last Section, $\nu=\theta^{\perp}$, and we propagate the boundary data $\varphi$ along the integral curves of $\theta^{\perp}$. Heuristically, $\theta$ is orthogonal to the level lines of $u$. Coming back to our general discussion, we want to construct a function $u: \Omega \rightarrow \mathbb{R}$ such that $\left.u\right|_{\partial \Omega}=\varphi$ and $u$ being constant along the integral curves of $\nu$, i.e., the solutions of the system of ordinary differential equations

$$
\frac{d X}{d t}=\nu(X)
$$

This amounts to say that

$$
\nu \cdot \nabla u=0
$$

a first order transport equation whose characteristic curves are the solution of the system (18). Let us discuss the difficulties posed by this formulation. First of all, existence and uniqueness of solutions of (18) is guaranteed when $\nu$ is a Lipschitz vector field, a very strong regularity assumption, which excludes any singularity for $\nu$. More general existence results have been obtained in [13] via the study of transport equations, indeed, via formulations analog to (19). Typically, they are assuming that $\nu$ is in some Sobolev space like $\in W_{l o c}^{1,1}\left(\mathbb{R}^{N}\right)$, with some other integrability assumptions, and $\operatorname{div}(\nu) \in L^{\infty}\left(\mathbb{R}^{N}\right)$. These results have been further extended in [12], [30]. In particular, P.L. Lions in [30] proves a.e. existence of solutions of (18) for vector fields which are piecewise in $W^{1,1}$ in a precise sense defined by the author. As observed in these papers, it is not known if the previous result is true for BV vector fields. On the other hand, even for a vector field in $W^{1,1}$, for which we have existence a.e. of solutions of (18), the problem of constructing $u$ satisfying (19) and such that $\left.u\right|_{\partial \Omega}=\varphi$ is not obvious. Indeed, consider a smooth vector field $\nu$ defined on a simple domain, like $D:=\left\{x \in \mathbb{R}^{2}:|x| \leq 1\right\}$ and suppose that the integral curves of $\nu$ are curves that foliate $D$ and such that at any point of $\partial D$ we start a curve that ends in another point of $\partial D$. Then the only possibility to extend $u$ to $\Omega$ so that $\left.u\right|_{\partial \Omega}=\varphi$ in a classical sense is that $\varphi$ takes the same values at the beginning and endpoints of the integral curves of $\nu$. A possibility to overcome this difficulty, would be to use the vanishing viscosity method, i.e., to solve the elliptic equation

$$
\nu \cdot \nabla u_{\epsilon}+\epsilon \Delta u_{\epsilon}=0
$$


and let $\epsilon \rightarrow 0$. Then, we hope the sequence $u_{\epsilon}$ to converge to some bounded function $u$ which solves the problem in a distributional sense. We do not have further information on the regularity of $u$. On the other hand we do not know in which sense the boundary conditions hold.

Let us consider the problem from the algorithmic point of view, i.e., we want to design an effective algorithm to solve it. Since the problem may be ill-posed, because of incompatibility of boundary data joining two integral curves of the vector field $\nu$, we propose a variational formulation of the problem. Let $\theta=\nu^{\perp}$. Assume that $|\theta| \leq 1$. If a solution exists, then $\theta$ should point in the normal direction to the level lines of $u$. We implicitly assume that $\theta$ should be constructed as the vector field normal to the level curves of $u$. Then, formally, $\theta \cdot \nabla u=|\nabla u|$. Thus, it seems reasonable to minimize the functional

$$
F(u)=\int_{\Omega}|\nabla u|-\int_{\Omega} \theta \cdot \nabla u
$$

(exactly the one introduced above) defined in the set of functions of bounded variation $B V(\Omega)$ whose trace at the boundary is given by $\varphi$. Let us formally integrate by parts in the second term of $F(u)$ to obtain

$$
F(u)=\int_{\Omega}|\nabla u|+\int_{\Omega} \operatorname{div}(\theta) \cdot u-\int_{\partial \Omega} \theta \cdot \vec{n} u,
$$

Since $u, \theta$ are known at the boundary, minimizing $F$ amounts to minimize

$$
E(u)=\int_{\Omega}|\nabla u|+\int_{\Omega} \operatorname{div}(\theta) \cdot u .
$$

Let us make precise the class of admissible functions where $E$ is minimized. We assume that $\operatorname{div}(\theta) \in L^{1}(\Omega)$ and $\varphi \in L^{\infty}(\partial \Omega)$. It seems reasonable to impose that the solution $u$ is a bounded function with an $L^{\infty}$ bound given by $\|\varphi\|_{\infty}$ (or a constant related to $\|\varphi\|_{\infty}$ and the size of $\Omega$ ). Then the second integral in the definition of $E(u)$ is well defined. The first integral requires the use of the space of bounded variation functions. Thus our admissible class is $\mathcal{A}=\left\{u \in B V(\Omega):|u(x)| \leq\|\varphi\|_{\infty}\right.$ a.e. $\left.\left.u\right|_{\partial \Omega}=\varphi\right\}$. We propose

$$
\underset{u \in \mathcal{A}}{\operatorname{Minimize}} \int_{\Omega}|\nabla u|+\int_{\Omega} \operatorname{div}(\theta) \cdot u
$$

As it is well known ([21], [15]) the solution of this problem has to be understood in a weak sense as the solution of the problem

$$
\begin{aligned}
& \text { Minimize } \int_{\Omega}|\nabla u|+\int_{\Omega} \operatorname{div}(\theta) \cdot u+\int_{\partial \Omega}|u-\varphi| d H^{1} \\
& u \in B V(\Omega) \\
& |u| \leq\|\varphi\|_{\infty} .
\end{aligned}
$$

Then we have the following result.

Theorem 1 Let $\theta \in L_{l o c}^{1}(\Omega)^{2}$, with $\operatorname{div}(\theta) \in L^{1}(\Omega), \varphi \in L^{\infty}(\partial \Omega)$. Then there is a function $u \in B V(\Omega)$ such that $|u(x)| \leq\|\varphi\|_{\infty}$ a.e. minimizing (22).

Proof. The result is contained in $([21])$, Theorem 1.4.

This clarifies the role of the term (17) in (10). 


\section{Numerical experiments}

To minimize (21) we use the steepest descent method. For that, we formally compute the Euler-Lagrange equation for $u$, namely,

$$
-\operatorname{div}\left(\frac{\nabla u}{|\nabla u|}\right)+\operatorname{div}(\theta)=0
$$

supplemented with Dirichlet boundary conditions for $u$. In practice, we use the evolution equation

$$
u_{t}=\operatorname{div}\left(\frac{\nabla u}{|\nabla u|}\right)-\operatorname{div}(\theta)
$$

with Dirichlet boundary data and initial condition constructed as an ad-hoc interpolation that will be corrected by the equation. General existence results which can be adapted to this case can be found in ([4]). Note that the vector field $\theta$ is assumed to be known in this case. This limits the usefulness of this model. But we present some experiments below to illustrate the role of this term.

To minimize the functional (10) we used the steepest descent method. For that, we formally compute the Euler-Lagrange equations for $(\theta, u)$. The equations for $\theta$ are

$$
\nabla_{\theta} E(\theta, u)=-p \nabla\left[(a+b|\nabla k * u|)|\operatorname{div}(\theta)|^{p-2} \operatorname{div}(\theta)\right]-\nabla u \chi_{\Omega}-\nabla u_{0} \chi_{B}=0 \quad \text { in } \tilde{\Omega} .
$$

The equation for $u$ is

$$
\nabla_{u} E(\theta, u)=-\operatorname{div}\left(k * g \frac{\nabla k * u}{|\nabla k * u|}\right)-\alpha \operatorname{div}\left(\frac{\nabla u}{|\nabla u|}\right)+\operatorname{div}(\theta)=0 \quad \text { in } \Omega,
$$

where $g=b|\operatorname{div}(\theta)|^{p}$. In our experiments, we take $k$ a Gaussian kernel with small variance, say one or two pixels. In practice, one can also dismiss the kernel $k$. These equations have to be complemented with the corresponding boundary conditions for $\theta$ and $u$ specified by the

admissible class, i.e., we specify the normal component of $\theta$ in $\partial \tilde{\Omega}$ and the Dirichlet boundary condition for $u$ in $\partial \Omega$, since $u=u_{0}$ in $B$. Thus, we solve the evolution problems

$$
\theta_{t}=-\nabla_{\theta} E(\theta, u)
$$

and

$$
u_{t}=-\nabla_{u} E(\theta, u)
$$

supplemented with the corresponding boundary data and initial conditions. The initial conditions are ad-hoc interpolations, for instance, we can take $u$ inside $\Omega$ as the average value of $u_{0}$ in $B, \theta$ inside $\Omega$ being the direction of the gradient of $u$. One can also take a geodesic propagation inside $\Omega$ of the values of $u_{0}$ in $B$, with $\theta$ being again the direction of the gradient of $u$. In the experiments below, this algorithm is used to interpolate level sets. In this, we follow the approach in [31], [32]. The image in $B$ is decomposed into level sets and we get a family of binary images $u_{0 \lambda}=\chi_{\left[u_{0} \geq \lambda\right]}, \lambda=0,1,2, \ldots, 255$. These functions are interpolated inside $\Omega$ and we obtain a family of level sets $X_{\lambda}$. Then the function $u$ is reconstructed using the reconstruction formula

$$
u(x)=\sup \left\{\lambda \in\{0,1, \ldots, 255\}: x \in X_{\lambda}\right\}
$$


With this approach, we diminish the diffusive effects of the above algorithm and we better capture the shapes and discontinuities on the interpolated image. The constraints on $\theta$ and $\|u\|_{\infty}$ can be introduced after each iteration of the above equations.

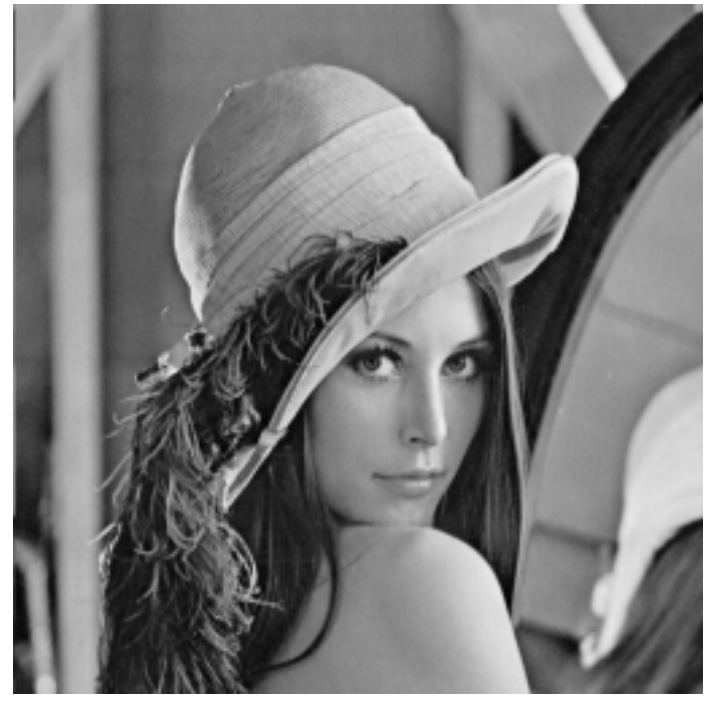

Figure 4.a.

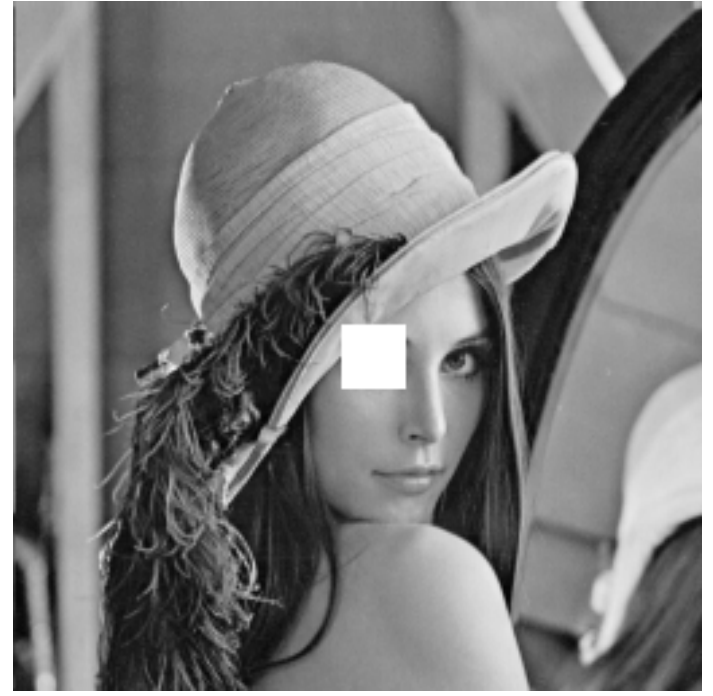

Figure 4.b.

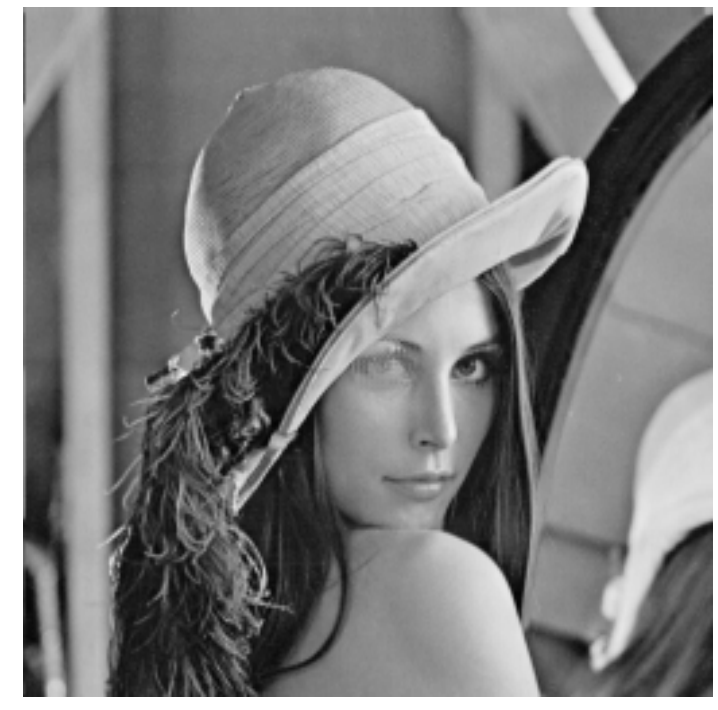

Figure 4.c.

Let us describe the experiments. First, in Figure 4 we display some experiment to illustrate functional (21). Figure 4.a displays the full image without the hole. Figure 4.b displays the image with the hole. The vector field $\theta$ has been computed on Figure 4.a and we see in Figure 4.c the result of interpolating the gray level knowing the vector field inside $\Omega$. We see that the shape of the eye is recovered but not the gray level. This is not a surprise since the gray level 
inside the eye cannot be recovered from the gray level on the boundary of $\Omega$. The algorithm is able to capture the shapes inside the eye by integrating the vector field $\theta$.

In the following experiments we show the results of the joint interpolation of gray level and the vector field of directions using functional (10). The experiments have been done with $p=1$ and/or $p=2$. The results are quite similar. Unless explicitly stated, we display the results obtained with $p=1$. Figure 5.a displays an image made of four circles covered by a square. In Figure 5.b we display the result of the interpolation. In Figure 6.a, we display an example where the hole is not simply connected. The interpolation is displayed in Figure 6.b. Figure 7.a is the image of Lena with two holes, a lower one in the hat and an upper one. Figure 7.b displays the result of the interpolation. Figure 8 displays a zoom of the region around the lower hole. In Figure 9.a we display a level set of $u_{0}$ corresponding to the region around the lower hole. Figures 9.b and 9.c display the corresponding interpolation with $p=1$ and $p=2$, respectively. Figure 10.a displays an image with text to be removed. Figure 10.b displays the corresponding reconstruction result. Figure 11.a displays a portion of an image with text. Figure 11.b displays the corresponding reconstruction result, obtained with $p=2$.

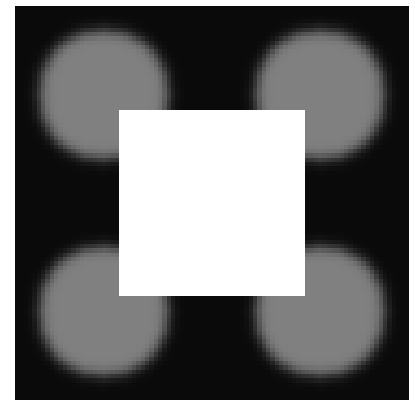

Figure 5.a.

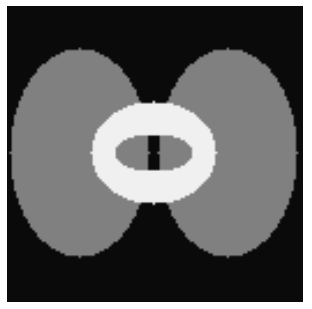

Figure 6.a.

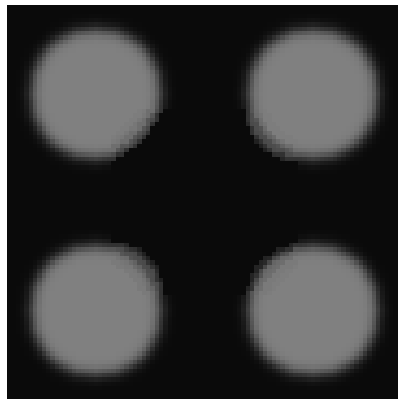

Figure 5.b.

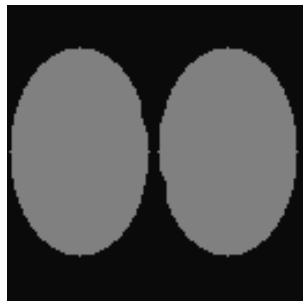

Figure 6.b. 


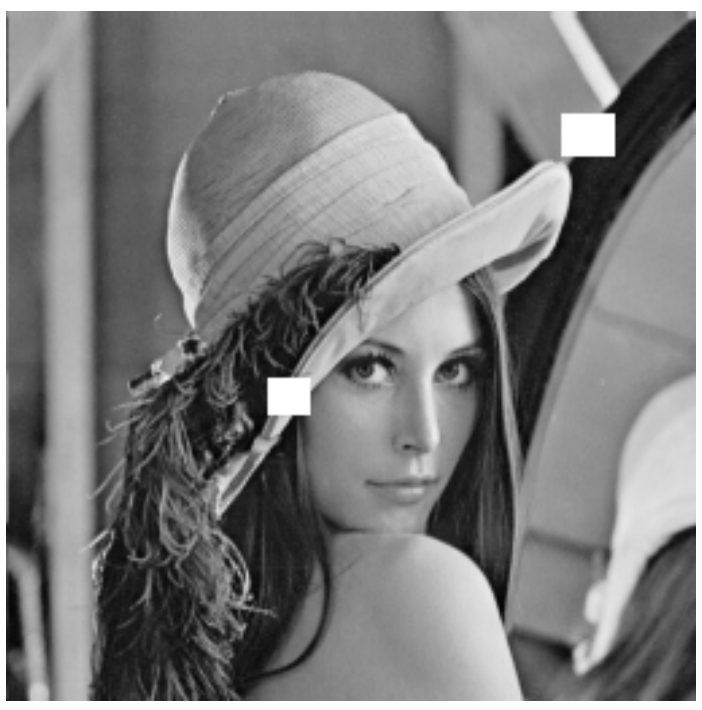

Figure 7.a.

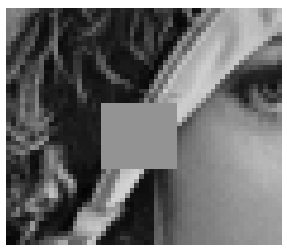

Figure 8.a.

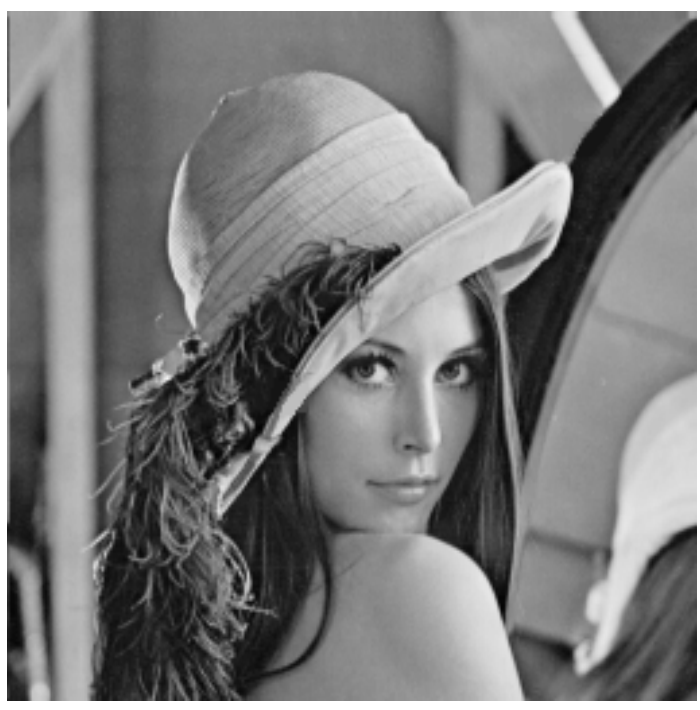

Figure 7.b.

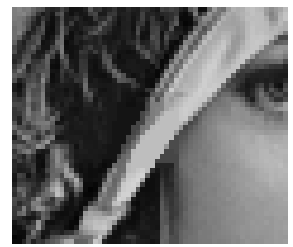

Figure 8.b.

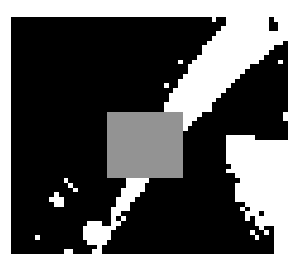

Figure 9.a.

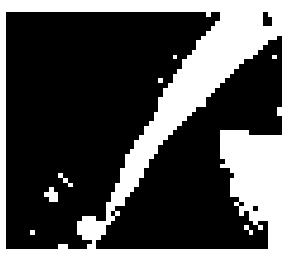

Figure 9.b.

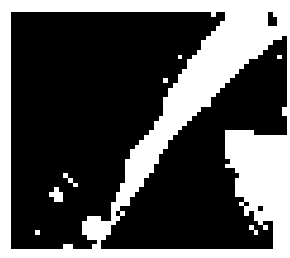

Figure 9.c. 


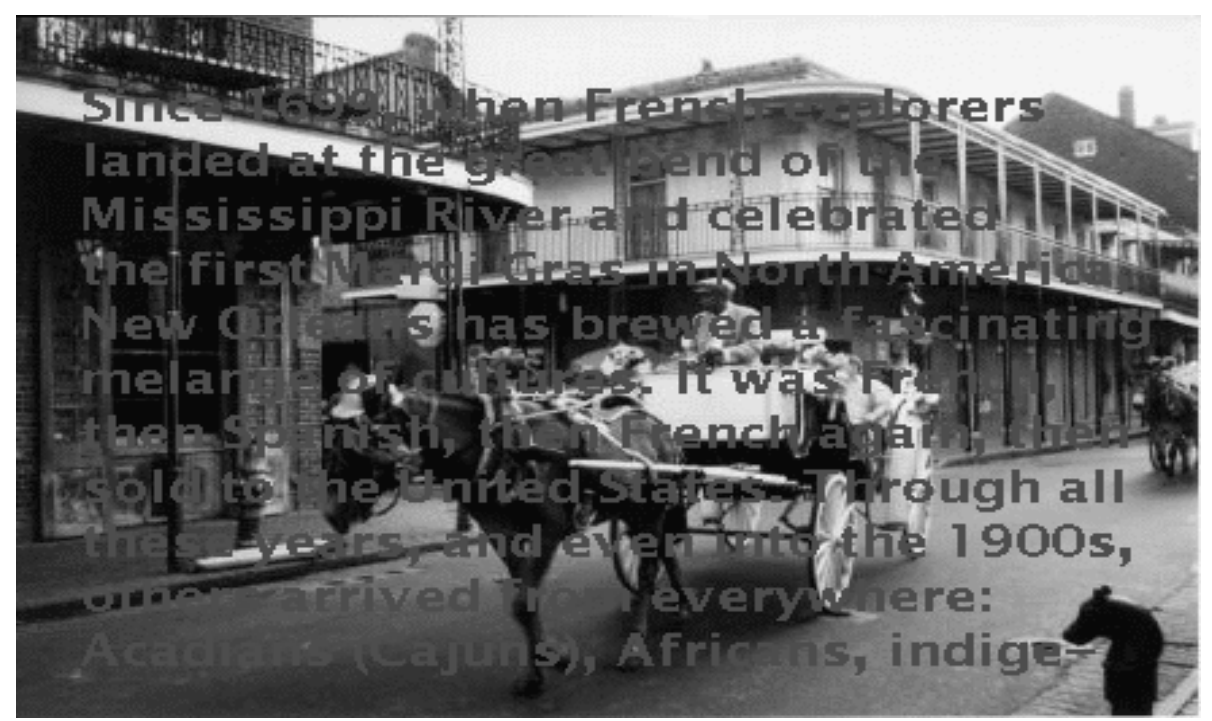

Figure 10.a.

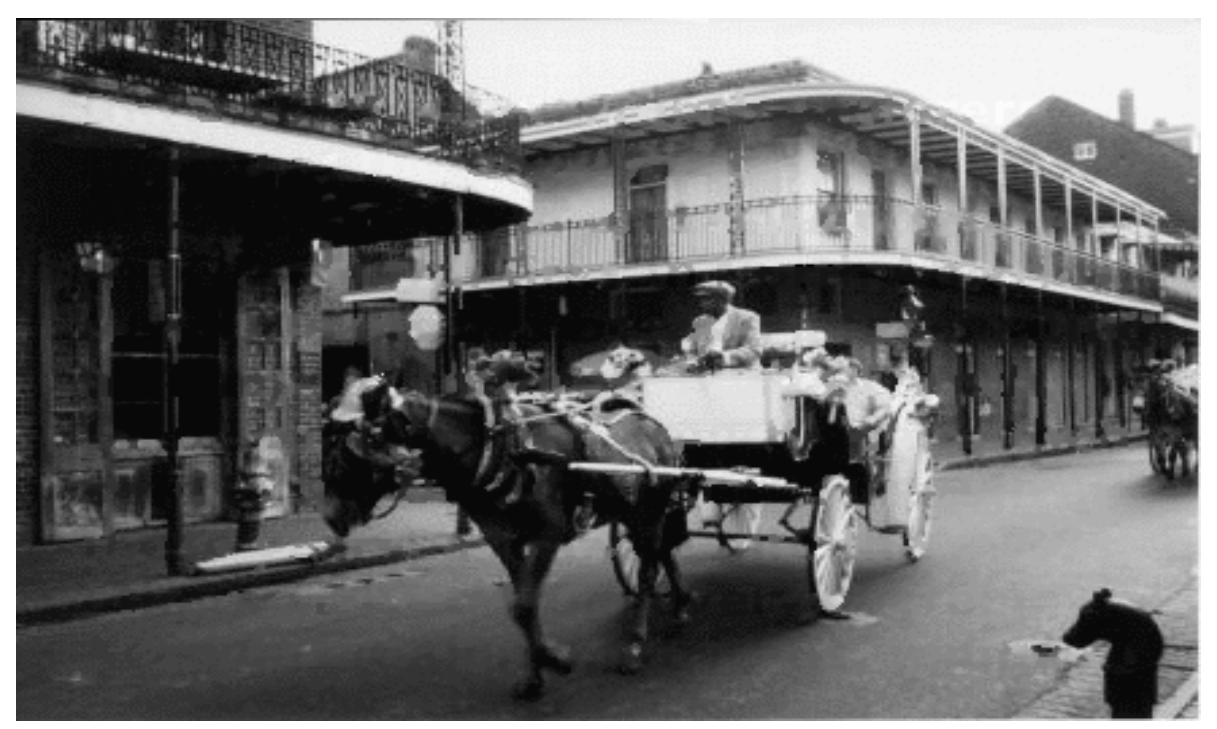

Figure 10.b.

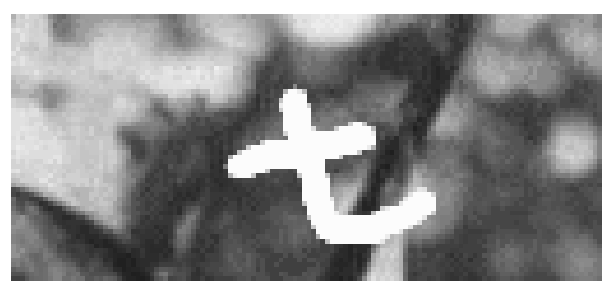

Figure 11.a.

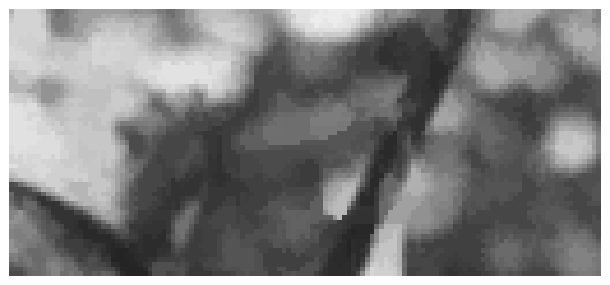

Figure 11.b. 


\section{Concluding remarks}

In this paper we have proposed a formal variational approach for filling-in regions of missing data in still images. The basic idea is to smoothly extend inside the hole both the vector field obtained from the image gradient and the corresponding gray values. We have presented a number of examples and showed theoretical results regarding the proposed formulation.

A number of research directions are suggested by the work here presented. First of all, we need to complement this algorithm by a technique capable of filling-in textured regions. Secondly, the extension of the framework to the filling-in of other type of missing imagery data is of great interest for a number of applications. Last, we would like to study these ideas for interpolation in video data. These topics will be the subject of subsequent reports.

\section{Appendix: Existence of minimizers}

Recall that $\tilde{\Omega}=\bar{\Omega} \cup B$ is an open bounded set whose boundary is Lipschitz. For simplicity, let us define the class $\mathcal{B}$ of admissible pairs $(\theta, u)$ where $\theta \in W^{1, p}(\operatorname{div}, \tilde{\Omega}), u \in B V\left(\tilde{\Omega}, B, u_{0}\right)$, $|\theta| \leq 1,|u| \leq\left\|u_{0}\right\|_{L^{\infty}(B)}$ and $\left.\theta \cdot n\right|_{\partial \tilde{\Omega}}=\left.\theta_{0} \cdot n\right|_{\partial \tilde{\Omega}}$.

Theorem 2 If $p>1$, there is a minimum $(\theta, u) \in \mathcal{B}$ for the problem (10).

Proof. Let us denote by $E(\theta, u)$ the energy defined in (10). Let $\left(\theta_{n}, u_{n}\right)$ be a minimizing sequence for $E(\theta, u)$. Since

$$
\int_{\tilde{\Omega}}\left|\nabla u_{n}\right|-\theta_{n} \cdot \nabla u_{n} \geq 0
$$

and $E\left(\theta_{n}, u_{n}\right)$ is bounded, we obtain that

$$
\int_{\tilde{\Omega}}\left|\operatorname{div}\left(\theta_{n}\right)\right|^{p}
$$

is bounded. Since $\left|\theta_{n}\right| \leq 1$, we have that $\theta_{n}$ is weakly relatively compact in all spaces $L^{q}(\tilde{\Omega})^{2}$ for all $1 \leq q<\infty$ and we may assume that $\theta_{n} \rightarrow \theta$ weakly in $L^{q}(\tilde{\Omega})^{2}$ for all $1 \leq q<\infty$ and in $W^{1, p}(\operatorname{div}, \tilde{\Omega})$. Now, integrating by parts the term $\int_{\tilde{\Omega}} \theta_{n} \cdot \nabla u_{n}$, we obtain

$$
\begin{aligned}
\int_{\tilde{\Omega}} \theta_{n} \cdot \nabla u_{n} & =-\int_{\tilde{\Omega}} \operatorname{div}\left(\theta_{n}\right) u_{n}+\int_{\partial \tilde{\Omega}}\left[\theta_{n}, n\right] u_{n} \\
& =-\int_{\tilde{\Omega}} \operatorname{div}\left(\theta_{n}\right) u_{n}+\int_{\partial \tilde{\Omega}}\left[\theta_{0}, n\right] u_{0}
\end{aligned}
$$

The integration by parts is possible by results of Anzellotti ([2]) given above. From the above identity, we obtain

$$
\left|\int_{\tilde{\Omega}} \theta_{n} \cdot \nabla u_{n}\right| \leq\left\|\operatorname{div}\left(\theta_{n}\right)\right\|_{p}\left\|u_{n}\right\|_{p^{\prime}}+\int_{\partial \tilde{\Omega}}\left|u_{0}\right|
$$

since $u_{n}=u_{0}$ in $\partial \tilde{\Omega}$, where $p^{\prime}$ is the exponent conjugated to $p$. Since we minimize the energy $E(\theta, u)$ for functions with an $L^{\infty}$ bound, we obtain that

$$
\left|\int_{\tilde{\Omega}} \theta_{n} \cdot \nabla u_{n}\right|
$$


is uniformly bounded in $n$. The consequence of this observation is that

$$
\int_{\tilde{\Omega}}\left|\nabla u_{n}\right|
$$

is also bounded. Then, modulo a subsequence, we may assume that $u_{n}$ converges to some function $u$ in $L^{1}(\tilde{\Omega})$. Note that $u \in B V\left(\tilde{\Omega}, B, u_{0}\right)$. Since we have an $L^{\infty}$ bound on $u_{n}$, we also have that $u_{n}$ converges to $u$ in $L^{q}(\tilde{\Omega})$ for all $1 \leq q<\infty$. Then $\nabla k * u_{n} \rightarrow \nabla k * u$ uniformly in $\tilde{\Omega}$. In particular, we obtain

$$
\int_{\tilde{\Omega}}|\operatorname{div}(\theta)|^{p}(a+b|\nabla k * u|) d x \leq \liminf _{n} \int_{\tilde{\Omega}}\left|\operatorname{div}\left(\theta_{n}\right)\right|^{p}\left(a+b\left|\nabla k * u_{n}\right|\right) d x
$$

and

$$
\int_{\tilde{\Omega}}|\nabla u| \leq \liminf _{n} \int_{\tilde{\Omega}}\left|\nabla u_{n}\right|
$$

Finally, since $\operatorname{div}\left(\theta_{n}\right)$ weakly converges to $\operatorname{div}(\theta)$ in $L^{p}(\tilde{\Omega})$ and $u_{n} \rightarrow u$ in $L^{p^{\prime}}(\tilde{\Omega})$, passing to the limit in

$$
\int_{\tilde{\Omega}} \theta_{n} \cdot \nabla u_{n}=-\int_{\tilde{\Omega}} \operatorname{div}\left(\theta_{n}\right) u_{n}+\int_{\partial \tilde{\Omega}}\left[\theta_{0}, n\right] u_{0}
$$

we get that $\int_{\tilde{\Omega}} \theta_{n} \cdot \nabla u_{n}$ converges to

$$
-\int_{\tilde{\Omega}} \operatorname{div}(\theta) u+\int_{\partial \tilde{\Omega}}\left[\theta_{0}, n\right] u_{0}=\int_{\tilde{\Omega}} \theta \cdot \nabla u .
$$

Thus, collecting all these facts, we obtain that

$$
E(\theta, u) \leq \liminf _{n} E\left(\theta_{n}, u_{n}\right) .
$$

The pair $(\theta, u)$ is a minimum of $E$ in the class of admissible functions for this functional.

\section{Acknowledgments}

We thank S. Betelu, A. Bertozzi, T. Chan, C. Kenney, P.L. Lions, J.M. Morel, S. Osher, E. Simoncelli, and J. Shen for interesting conversations on image inpainting and filling-in. This work was partially supported by a grant from the Office of Naval Research ONR-N00014-97-1-0509, the Office of Naval Research Young Investigator Award, the Presidential Early Career Awards for Scientists and Engineers (PECASE), a National Science Foundation CAREER Award, by the National Science Foundation Learning and Intelligent Systems Program (LIS), and the TMR European project "Viscosity Solutions and their applications," reference FMRX-CT98-0234.

\section{References}

[1] L. Ambrosio, N. Fusco and D. Pallara, Functions of Bounded Variation and Free Discontinuity Problems, Forthcoming book. 
[2] G. Anzellotti, "Pairings between measures and bounded functions and compensated Compactness," Ann. di Mat. Pura ed Appl. 135, 293-318, 1993.

[3] F. Andreu, C. Ballester, V. Caselles, J. M. Mazón, "Minimizing Total Variation flow," Differential and Integral Equations, to appear.

[4] F. Andreu, C Ballester, V. Caselles and J.M. Mazón, "The Dirichlet problem for the Total Variation flow," preprint.

[5] G. Bellettini, G. Dal Maso and M. Paolini, "Semicontinuity and relaxation properties of a curvature depending functional in 2D," Ann. Scuola Normale Sup. di Pisa, Cl. Sci. 20(4), pp. 247-297, 1993.

[6] M. Bertalmio, G. Sapiro, C. Ballester and V. Caselles, "Image inpainting," University of Minnesota IMA TR, December 1999 (available at www.ima.umn).

[7] M. Bertalmio, G. Sapiro, C. Ballester and V. Caselles, "Image inpainting," to appear in Computer Graphics, SIGGRAPH 2000, July 2000.

[8] C. Braverman. Photoshop retouching handbook. IDG Books Worldwide, 1998.

[9] T. Chan and J. Shen, "Mathematical models for local deterministic inpaintings," UCLA CAM Report 00-11, March 2000 (available at www.math.ucla.edu).

[10] R. Dautray and J.L. Lions, Analyse Mathématique et Calcul Numérique pour les Sciences et les Techniques, Tome 2, Masson, 1985.

[11] F. Demengel, Introduction aux Équations aux Derivées Partielles Elliptiques, Diderot Ed. 1999 .

[12] B. Desjardins, "A few remarks on ordinary differential equations," Comm. in Partial Diff. Equations 21, pp. 1667-1703, 1996.

[13] R. J. DiPerna and P. L. Lions, "Ordinary differential equations, transport theory and Sobolev spaces," Inventiones Math. 98, pp. 511-547, 1989.

[14] A. Efros and T. Leung, "Texture synthesis by non-parametric sampling," Proc. IEEE International Conference Computer Vision, pp. 1033-1038, Corfu, Greece, September 1999.

[15] I. Ekeland and R. Temam, Convex Analysis and Variational Problems, North Holland, Amsterdam, 1976.

[16] G. Emile-Male. The Restorer's Handbook of Easel Painting. Van Nostrand Reinhold, New York, 1976.

[17] L.C. Evans and R.F. Gariepy, Measure Theory and Fine Properties of Functions, Studies in Advanced Math., CRC Press, 1992. 
[18] G. P. Galdi, An Introduction to the Mathematical Theory of the Navier-Stokes Equations, Volume I, Springer Verlag, 1994.

[19] D. Heeger and J. Bergen. "Pyramid based texture analysis/synthesis," Computer Graphics, pp. 229-238, SIGGRAPH 95, 1995.

[20] P. G. de Gennes and J. Prost, The Physics of Liquid Crystals, Oxford Science Publications, 1998.

[21] M. Giaquinta, G. Modica and J. Soucek, "Functionals with linear growth in the calculus of variations I," Comment. Math. Univ. Carolina 20, pp. 143-156, 1979.

[22] A. Hirani and T. Totsuka. "Combining frequency and spatial domain information for fast interactive image noise removal," Computer Graphics, pp. 269-276, SIGGRAPH 96, 1996.

[23] L. Joyeux, O. Buisson, B. Besserer, S. Boukir. "Detection and removal of line scratches in motion picture films," Proceedings of CVPR'99, IEEE Int. Conf. on Computer Vision and Pattern Recognition, Fort Collins, Colorado, USA, June 1999.

[24] C. Kenney and J. Langan, "A new image processing primitive: Reconstructing images from modified flow fields," preprint, University of California, Santa Barbara.

[25] D. King. The Commissar Vanishes. Henry Holt and Company, 1997.

[26] R. Kohn and R. Temam, "Dual spaces of stresses and strains, with applications to Hencky plasticity," Appl. Math. Optimization 10 (1983), 1-35.

[27] A.C. Kokaram, R.D. Morris, W.J. Fitzgerald, P.J.W. Rayner. "Detection of missing data in image sequences," IEEE Transactions on Image Processing 11(4), pp. 1496-1508, 1995.

[28] A.C. Kokaram, R.D. Morris, W.J. Fitzgerald, P.J.W. Rayner. "Interpolation of missing data in image sequences," IEEE Transactions on Image Processing 11(4), pp. 1509-1519, 1995.

[29] A. Lichnewski and R. Temam, "Pseudosolutions of the time dependent minimal surface problem," Journal of Differential Equations 30, pp. 340-364, 1978.

[30] P.L. Lions, "Sur les équations différentielles ordinaires et les équations de transport," Comptes Rendus Acad. Sciences 326, pp. 833-838, 1998.

[31] S. Masnou, Filtrage et Desocclusion d'Images par Méthodes d'Ensembles de Niveau, Thèse, Université Paris-Dauphine, 1998.

[32] S. Masnou and J.M. Morel, "Level lines based disocclusion," 5th IEEE International Conference on Image Processing, Chicago, Illinois, October 4-7, 1998.

[33] M. Nitzberg, D. Mumford, and T. Shiota, Filtering, Segmentation, and Depth, SpringerVerlag, Berlin, 1993. 
[34] L. Rudin, S. Osher and E. Fatemi. "Nonlinear total variation based noise removal algorithms," Physica D 60, pp. 259-268, 1992.

[35] E. Simoncelli and J. Portilla. "Texture characterization via joint statistics of wavelet coefficient magnitudes," 5th IEEE Int'l Conf. on Image Processing, Chicago, IL. Oct 4-7, 1998 .

[36] D. Strong and T. Chan, "Exact solutions to Total Variation regularization problems," UCLA CAM Report 96-41, October 1996.

[37] R. Temam, "On the continuity of the trace of vector functions with bounded deformation," Appl. Analysis 11, pp. 291-302, 1993.

[38] S. Walden. The Ravished Image. St. Martin's Press, New York, 1985.

[39] W. P. Ziemer, Weakly Differentiable Functions, GTM 120, Springer Verlag, 1989. 